\title{
DUPLICATION DEFICIENCY HETEROZYGOTES IN AEDES AEGYPTI*
}

\author{
S. VED BRAT and K. S. RAI \\ Department of Biology, University of Notre Dame, Indiana 46556, U.S.A.
}

Received 13.iv.73

\begin{abstract}
SUMmary
Two types of duplication-deficiency heterozygotes have been discovered from a translocation stock of Aedes aegypti. The structural interchange involved one arm of chromosome 2 with chromosome 1 and the other arm with chromosome 3.

Both types of $d p-d f$ heterozygotes were deficient for a part of chromosome 2. But in one case a part of chromosome 1 and, in the other, a part of chromosome 3 was duplicated.

During meiosis, chromosomes with a duplication usually formed homomorphic ring bivalents in a variable frequency. The remaining four chromosomes formed chain multiples. Ring multiples were never observed.

In one heterozygote where chromosome 3 was involved in duplication, approximately 91 per cent of the cells showed a chiasma in one of the interstitial regions.

The frequency of the interstitial chiasma corresponded with the expected equational segregation during first anaphase of meiosis.
\end{abstract}

\section{IntRoduction}

IN interchange heterozygotes, gametes carrying duplications and deficiencies are produced from non-disjunctional segregation of adjacent chromosomes which form a multiple association during meiosis. They can also arise from crossing over in the interstitial segments of the translocated chromosomes. They are rarely known to be viable and produce progenies. They are reported to be effective in fertilisation in some animals (Lindsley and Grell, 1969) and plants. Catcheside (1963) found two types of duplication deficiency gametes in Oenothera which produced viable progenies. The present paper deals with a similar case which we have come across during our studies on a radiation induced male linked double translocation in Aedes aegypti. One of the heterozygotes also obtained a very high incidence of interstitial chiasma in one of the translocated chromosomes. Observations on the segregation of the chromosomes involved in the interstitial chiasma are also reported.

\section{Materials ANd methods}

Meiosis was studied from 12-18 hours old pupae belonging to a translocation stock designated T(1-2)i. All pupae but one with red eyes, were

This work received support from the Atomic Energy Commission Contract AT(11-1)-38 with the Radiation Laboratory, University of Notre Dame, Notre Dame, Indiana. This is AEC Document No. C00-38-893. This work was done while S. V. B. was on leave of absence from Botany Department, Panjab University, Chandigarh, India.

* Dedicated to Professor C. D. Darlington on his seventieth birthday. 
wild type for eye colour. The stock shows pseudolinkage between $M$-re (male determining factor and red eye) on chromosome 1, and $s$ (spot abdomen) on chromosome 2. Chromosome 3 marker blt (black tarsus) segregates independently but min (miniature) which is also present on chromosome 3 but on the opposite end of the linkage map III, is pseudolinked with $M$. Involvement of all the three chromosomes in the interchange was also confirmed from observations on meiosis when multiple associations of six chromosomes are formed.

The stock has been maintained in the laboratory by crossing the males showing pseudolinkage between $M$-re and $s$ with the multiple marker stock GV-RED, homozygous for respective alleles.

Pupae were dissected in distilled water. The testes were squashed in 2 per cent aceto-lacto-orcein. The slides were sealed with nail polish and stored in the refrigerator at $5-10^{\circ} \mathrm{C}$.

\section{Observations}

\section{(i) The structural change}

The standard complement of Aedes aegypti comprises three chromosomes. The smallest and the largest are metacentric and designated 1 and 2 respectively. Chromosome 3 is submetacentric and intermediate in size between the first two (McDonald and Rai, 1970). The karyotype of the interchange heterozygote has a distinct change in morphology of chromosome 1 and 2. The translocated chromosome $1,\left(1^{\prime}\right)$ and $2,\left(2^{\prime}\right)$ have become submetacentric (text-fig. 1). Both arms of $2^{\prime}$ have been involved in interchange, the short arm with $1^{\prime}$ and the long arm with $3^{\prime}$. Consequently, four interstitial regions $a, b, c, d$ have arisen.

Thus, crossing over in the interstitial region $a$ or $d$ can produce gametes carrying duplication for a part of chromosome 1 or 3 respectively and deficiency for a part of chromosome 2 (text-fig. 1). Similarly, the products of crossing over in the regions $c$ or $d$ would involve duplication for a part of chromosome 2 and deficiency for a part of 1 or 3 respectively. We have found heterozygotes belonging to the former category which arose from crossing over in the interstitial segments $a$ and $d$, and consequently possess deficiency for a part of chromosome 2 and duplication for a part of chromosome 1 and 3 respectively.

\section{(ii) Meiosis}

Chromosome behaviour both in the duplication-deficiency $(d p-d f)$ and interchange heterozygotes is expected to be similar in some respects. But, there are some distinct characters which differentiate one from the other. In a situation such as the one we have observed, chain multiples can be formed in both, whereas the ring multiples comprising six chromosomes can be formed only in the interchange heterozygotes. The $d p-d f$ heterozygote can instead form a homomorphic ring bivalent accompanied by a chain multiple of four chromosomes. The two chromosomes forming a homomorphic bivalent, can also obtain a multiple chiasma with another chromosome owing to the duplication present. These configurations can be formed subject to the condition that chiasmata remain confined to the pairing regions. 


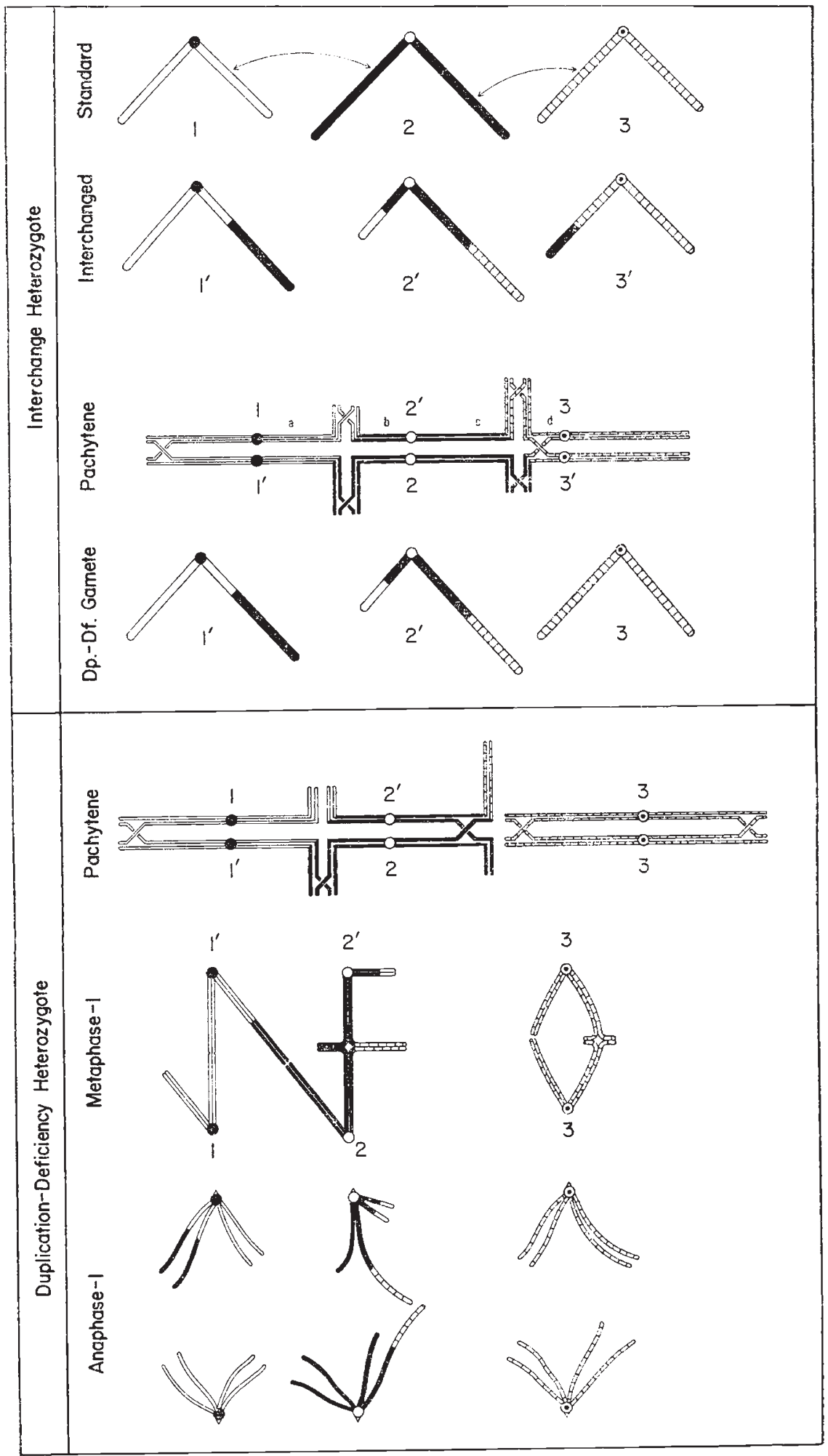

Fig. 1.-Model explaining the origin of duplication-deficiency heterozygote owing to the formation of interstitial chiasma in chromosome 3 in the interchange heterozygote. Also, the expected metaphase- 1 configuration with interstitial chiasma in the long arm of chromosome $2^{\prime}$ and the consequent equational segregation at A-1 in the duplicationdeficiency heterozygote. 
Observations on metaphase-1 revealed that all the 14 individuals obtained multiple associations of 3-6 chromosomes (table 1). Ring multiples were very rarely observed. In seven individuals chain multiples of 3-4 chromosomes accompanied by homomorphic bivalents were also observed. The frequency of the homomorphic ring bivalents was however variable. These individuals have been considered to be $d p$ - $d f$ heterozygotes. Two amongst them, including one with red eyes, had a duplication for a part of chromosome $1\left(d p_{1}-d f_{2}\right)$ and the remaining five had a duplication for a part of chromosome $3\left(d p_{3}-d f_{2}\right)$, whereas all had a deficiency for a part of chromosome 2 (table 1).

TABLE 1

Metaphase-1 associations in 14 individuals belonging to three categories from a sample of $T(1-2) i$

Serial No.

Associations

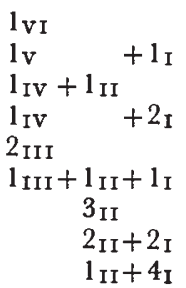

Total
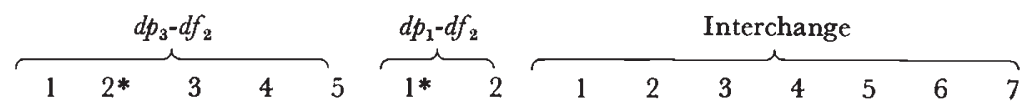

$$
\begin{array}{rrrrrrr}
- & - & - & 3 & 5 & - & 1 \\
2 & - & 1 & 3 & 1 & - & 1 \\
6 & 10 & 2 & 11 & 5 & 2 & 14 \\
- & - & - & - & 1 & - & - \\
- & - & - & - & - & - & - \\
7 & 18 & 24 & 1 & - & 11 & 2 \\
3 & 8 & 1 & 2 & - & 1 & 1 \\
7 & 22 & - & - & - & 4 & - \\
1 & - & - & - & - & - & - \\
\hline 26 & 58 & 28 & 20 & 12 & 18 & 19
\end{array}
$$

$\begin{array}{rrrrrrr}1 & 8 & 3 & 5 & 16 & 2 & 1 \\ - & 2 & 1 & - & 4 & 1 & - \\ - & 25 & 25 & 15 & 16 & 8 & 6 \\ - & - & 1 & - & 2 & - & 1 \\ 2 & - & 1 & 1 & 1 & - & - \\ 13 & 4 & 11 & 3 & 6 & 1 & - \\ - & 5 & 3 & 3 & 3 & 2 & 1 \\ - & 1 & - & - & - & 1 & - \\ - & - & 1 & - & - & - & - \\ 16 & 45 & 46 & 27 & 48 & 15 & 9\end{array}$

Chiasmata per cell

$\begin{array}{lllll}3 \cdot 19 & 3.26 & 3.29 & 4 \cdot 15 & 4 \cdot 67\end{array}$

$3 \cdot 78 \quad 4 \cdot 11$

$\begin{array}{lllllll}3.25 & 4.02 & 4.36 & 4.44 & 4.79 & 5.00 & 5.78\end{array}$

* Referred to in the text.

(iii) $D p_{3}-d f_{2}$

One individual was studied in detail from 58 cells at metaphase- 1 of meiosis. In one cell, pairing of three chromosomes owing to the duplication was also observed at diplotene (plate I, fig. a). Due to the changed morphology of the translocated chromosomes and the formation of an interstitial chiasma in segment $c$ (text-fig. 1), it was possible to establish their identity in multiple associations at metaphase-1. Twenty-eight cells obtained chain multiples of three or four chromosomes. In all multiples, chromosome $2^{\prime}$ occupied one of the terminals of the chain (plate I, figs. b-f). Its short arm was usually achiasmate whereas the long arm more often formed an interstitial chiasma with chromosome 2. Thus, a chain multiple of four chromosomes often showed one chiasma each in the pairing segments $1-1^{\prime}$ and 1'-2 and the third chiasma in the interstitial segment 2-2' designated as $c$ (text-fig. 1, plate I, figs. b-d). When a chiasma failed to form in the pairing region 1-1', chromosome 1 was observed as a univalent (plate I, fig. f). In approximately 75 per cent of the cells, chromosome 3 formed a homomorphic ring bivalent (plate I, figs. b, c, f-h), irrespective of the fact that the remaining four chromosomes formed a multiple or not. Thus, 48.62 per cent of the total chiasmata were obtained only by chromosome 3 in this individual. Chiasma formation in the pairing segment $2^{\prime}-3$ and consequently, a multiple association involving chromosome 3 , was observed only twice (plate I, 


\section{Plate I}

Meiosis in duplication-deficiency heterozygote

(All figures are $\times 2700$ )

FIG. a.-Diplotene showing chromosome $2^{\prime}$ and both 3 having undergone pairing.

FIG. b.-Metaphase-I of meiosis with chromosome 3 forming a ring bivalent. The multiple involves $2^{\prime}-2-I^{\prime}-1$ with an interstitial chiasma between $2^{\prime}$ and 2 and shows alternate orientation.

FIG. c.-Two cells at metaphse-I of meiosis. One showing a chain multiple of four and a bivalent as in fig. $b$ and the other with two bivalents and two univalents.

FIG. d.-Chain multiple of four with adjacent orientation.

FIG. e.-Multiple of four involving chromosome 3 and showing chiasma both in the pairing and interstitial segments of long arm of chromosome $2^{\prime}$.

Fig. f.-Chain multiple of three and chromosome I and 3 forming a univalent and bivalent respectively.

Fig. g.-Metaphase-I with three bivalents, and chiasma in the interstitial region of $2^{\prime}$ and pairing region of $I^{\prime}-1$.

FIG. h.-Metaphase-1 with two bivalents and two univalents.

FIG. i.- Anaphase-I with equational segregation of the long arm of chromosome $2^{\prime}$ owing to interstitial chiasma.

FIG. j.-Two cells, one at beginning of anaphase showing equational segregation of long arm of chromosome $2^{\prime}$; the second at metaphase-2 showing non-disjunction of chromosome $2^{\prime}$ and $I$, besides equational segregation of $2^{\prime}$ at anaphase- $I$.

FIG. k.-Metaphase-2 showing inequality of sister chromatids due to crossing over in the interstitial region of the long arm of chromosome $2^{\prime}$. 

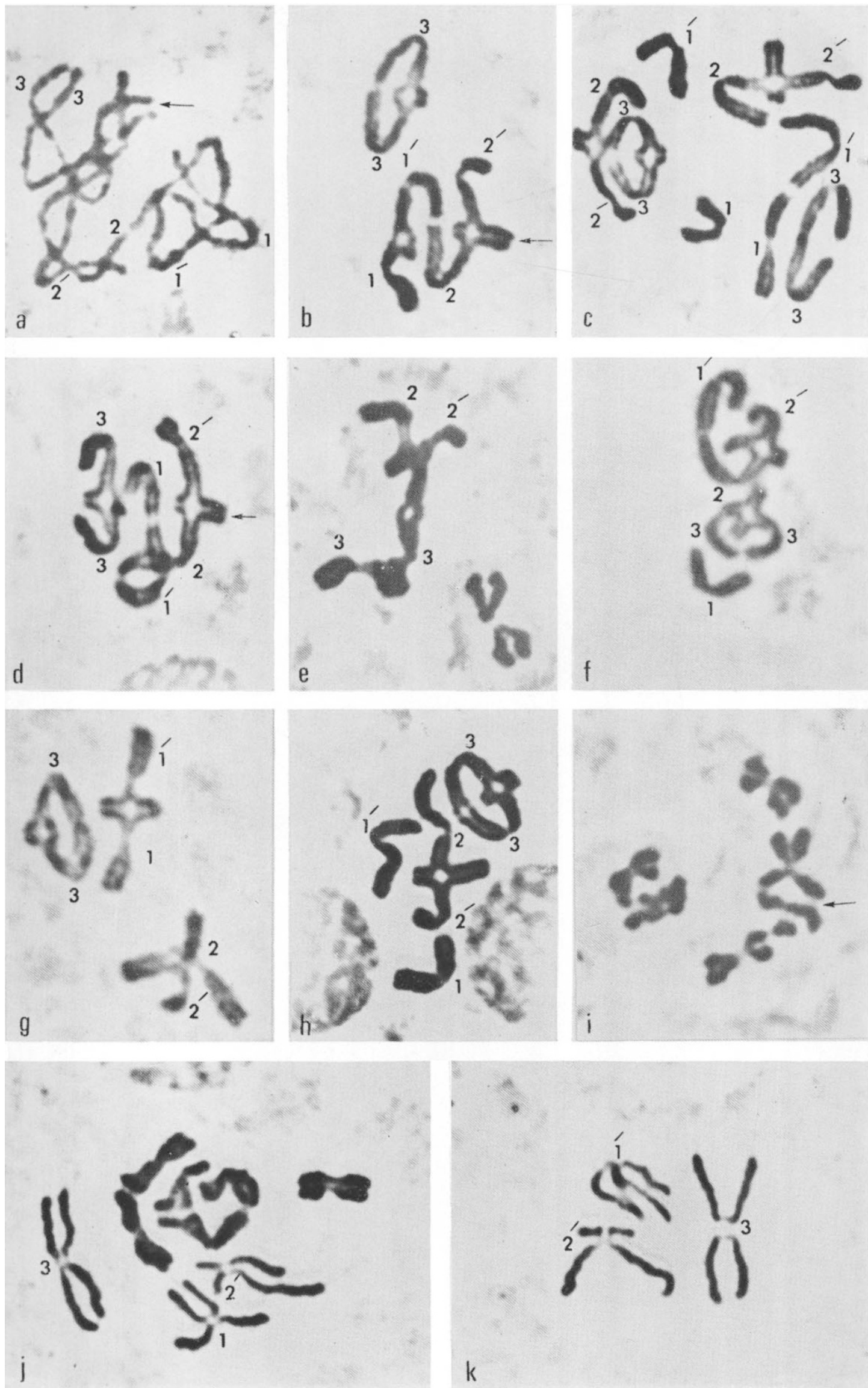
fig. e). Thus, it confirmed the homology of the terminal part of the long arm of chromosome 2' with that of chromosome 3. The pattern of chromosome pairing described here, i.e. chain multiples of 3 or 4 chromosomes accompanied by a homomorphic ring bivalent and also occasional chiasma formation between chromosome $2^{\prime}$ and 3 , strongly suggests that the individual consideration is a $d p_{3}-d f_{2}$ heterozygote which has arisen from a heterozygote with a double interchange (text-fig. 1 ).

\section{(iv) $D p_{1}-d f_{2}$}

In the case of the red-eyed individual which had arisen from crossing over in chromosome 1 between $r e$ and $M$ locus, 18 cells were examined (table 1 ). The general pattern of chiasma formation was similar to the one described above. Chain multiples of $3-4$ chromosomes were observed in 13 cells. In all, 12 cells showed homomorphic ring bivalents formed by chromosome 1 . The chain multiples were formed by chromosome 2', 3, 3 and 2, the latter at times also forming a univalent. The heterozygote evidently carried a duplication for a part of chromosome 1 and a deficiency a part of chromosome 2.

\section{(v) Crossing over in the interstitial region 2-2'}

In the $d p_{3}-d f_{2}$ heterozygote, approximately 91 per cent of the cells had interstitial chiasma in the long arm of chromosome 2' (text-fig. 1, plate I, figs. b-h). This was in strong contrast to the chiasma formation in any other interstitial region and also in any other individual in the sample. It is, however, similar to an interchange heterozygote of Chorthippus brunneus (John and Hewitt, 1963), where extensive chiasma formation in one of the interstitial segments has been reported. In the present case, the centromeres of chromosome 2 and 2 ' showed co-orientation facilitating anaphase-1 segregation of the two chromosomes to the opposite poles. Thus, the consequence of the interstitial chiasma could be correspondingly observed at anaphase-1 or metaphase-2 (text-fig. l, plate I, figs. i-k) when two sister chromatids showed inequality owing to the equational segregation of the chiasmate arms of chromosome 2 and $2^{\prime}$. The frequency of interstitial chiasma and that of equational segregation at anaphase-l showed a one to one co-relation (table 2). This is precisely expected according to the partial chiasmatype theory (Darlington, 1937) to which the present findings lend further support. This is also in accordance with the earlier findings on Lillium, Allium and Acrida, etc. (see Whitehouse, 1969), where heteromorphic bivalents have been used. for similar studies.

TABLE 2

Frequency of crossing-over and segregation of long arm of chromosome 2"

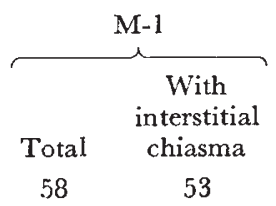

\begin{tabular}{|c|c|c|c|}
\hline \multicolumn{4}{|c|}{ A-1 } \\
\hline Total & $\begin{array}{c}\text { With } \\
\text { equational } \\
\text { segregation }\end{array}$ & Expected & Observed \\
\hline 74 & 63 & $91 \cdot 38 \%$ & $85 \cdot 13 \%$ \\
\hline
\end{tabular}




\section{REFERENCES}

GATCHESIDE, D. G. 1963. Nondisjunction in an Oenothera interchange heterozygote. Heredity, $18,63-75$.

DARLINGtoN, C. D. 1937. Recent Advances in Cytology. Churchill, London,

JOHN, B., AND HEWITT, G. M. 1963. A spontaneous interchange in Chorthippus brunneus with extensive chiasma formation in an interstitial segment. Chromosoma, 14, 638-650.

LiNDSLEY, D. L., AND Greld, E. H. 1969. Spermiogenesis in Drosophila. Genetics, 61 (Suppl.), 69-78.

MCDONALD, P. T., AND RAI, K. s. 1970. Correlation of linkage groups with chromosomes in the mosquito, Aedes aegypti. Genetics, 66, 475-485.

whitehouse, H. L. K. 1969. Towards an Understanding of the Mechanism of Heredity. Edward Arnold, London. 